\title{
Order of the Chiral and Continuum Limits in Staggered Chiral Perturbation Theory
}

\author{
C. Bernard \\ Department of Physics, Washington University, St. Louis, MO 63130, USA
}

(Dated: December 6, 2018)

\begin{abstract}
Dürr and Hoelbling recently observed that the continuum and chiral limits do not commute in the two dimensional, one flavor, Schwinger model with staggered fermions. I point out that such lack of commutativity can also be seen in four-dimensional staggered chiral perturbation theory $(\mathrm{S} \chi \mathrm{PT})$ in quenched or partially quenched quantities constructed to be particularly sensitive to the chiral limit. Although the physics involved in the $\mathrm{S} \chi \mathrm{PT}$ examples is quite different from that in the Schwinger model, neither singularity seems to be connected to the trick of taking the $\mathrm{n}^{\text {th }}$ root of the fermion determinant to remove unwanted degrees of freedom ("tastes"). Further, I argue that the singularities in $\mathrm{S} \chi \mathrm{PT}$ are absent in most commonly-computed quantities in the unquenched (full) QCD case and do not imply any unexpected systematic errors in recent MILC calculations with staggered fermions.
\end{abstract}

PACS numbers: 12.39.Fe, 12.38.Gc, 11.10.Kk 


\section{INTRODUCTION}

The one-flavor Schwinger model in two dimensions (2D) has recently been studied by Dürr and Hoelbling [1] using both overlap and staggered fermions. The square root of the staggered determinant is used to eliminate the extra taste degree of freedom. This is basically the same trick that is employed in current MILC three-flavor QCD simulations [2, 3, 4, 5] (the fourth root is required in 4D). Although many of the results in Ref. [1] are encouraging for the use of "rooted" staggered quarks, there is one disturbing feature: The chiral and continuum limits of $\langle\bar{\psi} \psi\rangle$ do not commute. If the continuum limit is taken first, the known continuum result is reproduced. But if the chiral limit is first taken for the staggered quarks, the continuum limit then disagrees with the exact result. This leads to three key questions:

- Do similar singularities appear in 4D QCD with staggered fermions?

- If they do appear, do such singularities induce uncontrolled systematic errors in the results reported in, e.g., Refs. [2, 3, 4, 5]?

- Are the singularities the result of the rooting procedure?

Here, I address these questions for 4D QCD in the context of staggered chiral perturbation

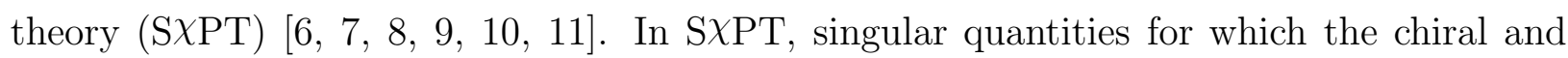
continuum limits fail to commute can easily be found in the quenched or partially quenched cases. As explained in Sec. III the way the noncommutativity comes about is simple: Taste violations split the masses of mesons, and the splitting becomes the dominant effect in the meson masses as the chiral limit is approached. When the physics is particularly sensitive to the chiral regime, the chiral and continuum limits do not commute. Quenching or partial quenching leads to such sensitivity through double poles in neutral meson propagators, which enhance the infrared (IR) regime in loop diagrams. In the context of S $\chi \mathrm{PT}$, the lack of commutativity has nothing directly to do with the issue of taking the root of the staggered determinant, and occurs in normal, "unrooted," staggered theories also.

In Sec. III I discuss the issue of commutativity of limits for unquenched S $\chi \mathrm{PT}$ ("full S $\chi$ PT"). Defining safe quantities as those for which the chiral and continuum limits commute, I argue that most standard physical quantities are safe because the full theory has no double poles and is therefore better behaved in the IR. In this sense it is very similar to full 
continuum chiral perturbation theory $(\chi \mathrm{PT})$. The safe quantities include those commonly determined in simulations, such as $f_{\pi}$ or the ratio of squared Goldstone meson mass to quark mass. However, the shorthand statement "standard quantities are safe" is misleading, since is in fact simple to write down other quantities for which the limits do not commute in full $\mathrm{S} \chi \mathrm{PT}$. A rough guide is that quantities that are finite in the chiral limit of full, continuum $\chi \mathrm{PT}$ are likely to be safe in this sense, although this guide itself has exceptions. Furthermore, there is at this point no proof, but only an intuitive argument for why the singularities are absent to all orders for the standard quantities in the full case. However, as discussed in Sec. IV] even if one assumes that the argument breaks down and singularities actually appear at some higher order, the associated new errors in previously computed quantities would be negligible.

Finally, in Sec. D. I mention two other examples of lack of commutativity, one with Wilson fermions and one in the quenched 2D Schwinger model. I point out that the known examples seem to indicate that the rooting procedure and the commutativity issues are independent. I conclude with a discussion of whether noncommutativity might be seen in topological and $\eta^{\prime}$ physics in $4 \mathrm{D}$.

\section{EXAMPLES FROM S $\chi$ PT}

The simplest example of noncommutativity of limits in S $\chi$ PT occurs in the quenched pion mass, where the "pion" is made of two degenerate valence quarks of mass $m_{V}$. In continuum quenched $\chi \mathrm{PT}$, we have at next-to-leading order (NLO) [12]:

$$
\left(\frac{M_{\pi}^{2}}{2 \mu m_{V}}\right)_{\text {cont }}=1+\frac{1}{16 \pi^{2} f^{2}} \frac{-2 m_{0}^{2}}{3} \ln \left(\chi_{V V} / \Lambda\right)+\cdots
$$

where $\Lambda$ is the chiral scale; $m_{0}^{2}$, the contribution to the $\eta^{\prime}$ mass from the anomaly; $f$, the decay constant normalized so that $f \approx 131 \mathrm{MeV}$; and $\chi_{V V}$, the tree-level pion mass squared, $\chi_{V V}=2 \mu m_{V}$. ( $\mu$ is a constant with dimensions of mass. $)$ The $\cdots$ represents less singular terms that do not lead to noncommutativity of limits. Here, and throughout this paper, I work in the infinite volume limit for simplicity.

The corresponding expression for the Goldstone pion in $\mathrm{S} \chi \mathrm{PT}$ is [7]

$$
\left(\frac{M_{\pi}^{2}}{2 \mu m_{V}}\right)_{\text {stag }}=1+\frac{1}{16 \pi^{2} f^{2}} \frac{-2 m_{0}^{2}}{3} \ln \left(\chi_{V V}^{I} / \Lambda\right)+\cdots,
$$


where $\chi_{V V}^{I}$ is the squared tree-level mass of the taste-singlet pion,

$$
\chi_{V V}^{I}=\chi_{V V}+a^{2} \Delta_{I}=2 \mu m_{V}+a^{2} \Delta_{I}
$$

with $a^{2} \Delta_{I}$ the splitting of the taste-singlet pion from the Goldstone pion. In Eq. (2) the neglected terms include effects of the taste-violating hairpins [7]. These are suppressed by $a^{2}$ and cause no problems with the limits.

Taking the ratio of lattice to continuum results, we have, to this order,

$$
\frac{\left(M_{\pi}^{2} / m_{V}\right)_{\mathrm{stag}}}{\left(M_{\pi}^{2} / m_{V}\right)_{\mathrm{cont}}}=1+\frac{1}{16 \pi^{2} f^{2}} \frac{-2 m_{0}^{2}}{3} \ln \left(1+\frac{a^{2} \Delta_{I}}{\chi_{V V}}\right)+\cdots .
$$

This ratio clearly goes to 1 if we take the continuum limit, $a \rightarrow 0$, first. But it blows up if the chiral limit $\left(m_{V} \rightarrow 0, \chi_{V V} \rightarrow 0\right)$ is taken first, because the staggered theory gives the wrong answer in this case. Of course $\chi \mathrm{PT}$ breaks down once the correction term gets large. In this case the breakdown occurs because of the "quenched chiral log" in the continuum. The logarithm is cut off in the staggered theory by the taste splitting, $a^{2} \Delta_{I}$.

This quenched example makes my basic point, but it is not very similar to the effect seen in Ref. [1]. There, the staggered theory is found to give a finite result for either order of the limits $m_{V} \rightarrow 0$ and $a \rightarrow 0$; it is just that the result is incorrect (different from the continuum) when $m_{V} \rightarrow 0$ is taken first. An example that has this kind of behavior can be found in the partially quenched $4 \mathrm{D}$ theory. ${ }^{1}$

For simplicity, I take $N_{S}$ degenerate sea quarks of mass $m_{S}$ in the partially quenched theory, and again consider a pion made from degenerate valence quarks of mass $m_{V}$. In the continuum, the pion mass at NLO is [13]

$$
\left(\frac{M_{\pi}^{2}}{2 \mu m_{V}}\right)_{\mathrm{cont}}=1+\frac{1}{16 \pi^{2} f^{2}} \frac{2}{N_{S}}\left(2 \chi_{V V}-\chi_{S S}\right) \ln \left(\chi_{V V} / \Lambda\right)+\cdots,
$$

where $\chi_{S S}=2 \mu m_{S}$, and $\cdots$ represents analytic terms.

In the staggered case, consider $N_{F}$ degenerate dynamical staggered fields with mass $m_{S}$. I define $N_{S}$ here as the number of sea quarks in the continuum limit: $N_{S}=N_{F}$ if the fourth root of the determinant is taken; while $N_{S}=4 N_{F}$ if it is not. The result has the same form when written in terms of $N_{S}$, whether or not the root is taken. Putting sea and valence

\footnotetext{
${ }^{1}$ But note that the example in Ref. 1] occurs in the full 2D theory.
} 
quarks separately degenerate in Eq. (48) of Ref. 7], one easily arrives at the one-loop mass of the Goldstone pion:

$$
\left(\frac{M_{\pi}^{2}}{2 \mu m_{V}}\right)_{\mathrm{stag}}=1+\frac{1}{16 \pi^{2} f^{2}} \frac{2}{N_{S}}\left(2 \chi_{V V}^{I}-\chi_{S S}^{I}\right) \ln \left(\chi_{V V}^{I} / \Lambda\right)+\cdots,
$$

where $\chi_{S S}^{I}$ is defined analogously to $\chi_{V V}^{I}$, Eq. (3), and $\cdots$ represents analytic terms and the effects of taste-violating hairpins, which again cause no problem with the limits.

If the continuum limit is taken first, Eq. (6) reproduces Eq. (15), so the two will give identical results no matter how the chiral limit is subsequently taken. On the other hand, if first we take the valence chiral limit $\left(m_{V} \rightarrow 0\right.$, with $m_{S}$ fixed), the lack of commutativity of limits exactly parallels the quenched case, with $\chi_{S S}$ playing the role of $m_{0}^{2}$. As before, $\mathcal{O}\left(a^{2}\right)$ terms in the staggered theory cut off a chiral log that is divergent in the continuum theory.

For my purposes, a more interesting chiral limit occurs when $m_{V}$ and $m_{S}$ both approach 0 , but with $m_{S}$ vanishing much more slowly, so that

$$
\chi_{S S} \sim \frac{-C}{\ln \left(\chi_{V V} / \Lambda\right)}
$$

as $\chi_{V V} \rightarrow 0$, with $C$ a positive constant. In this limit (with $a$ fixed in the staggered case)

$$
\begin{aligned}
& \left(\frac{M_{\pi}^{2}}{2 \mu m_{V}}\right)_{\mathrm{cont}} \rightarrow 1+\frac{1}{16 \pi^{2} f^{2}} \frac{2}{N_{S}} C \\
& \left(\frac{M_{\pi}^{2}}{2 \mu m_{V}}\right)_{\mathrm{stag}} \rightarrow 1+\frac{1}{16 \pi^{2} f^{2}} \frac{2}{N_{S}} a^{2} \Delta_{I} \ln \left(a^{2} \Delta_{I} / \Lambda\right)+\cdots
\end{aligned}
$$

where $\cdots$ represents additional $\mathcal{O}\left(a^{2} \ln \left(a^{2}\right)\right)$ or $\mathcal{O}\left(a^{2}\right)$ terms, coming from taste-violating hairpins or taste-violating analytic terms. If we now take the continuum limit,

$$
\left(\frac{M_{\pi}^{2}}{2 \mu m_{V}}\right)_{\text {stag }} \rightarrow 1
$$

in disagreement with Eq. (8) . On the other hand, the staggered theory clearly reproduces Eq. (8) if the continuum limit is taken first in Eq. (6).

Note that the noncommutativity of limits has nothing to do with the fourth-root prescription, and has exactly the same form with or without the fourth root. Of course, for fixed number $N_{F}$ of dynamical staggered fields, the effective number of continuum sea quarks $N_{S}$ depends on whether the root is taken, so we could say in that sense that the rooting prescription trivially affects the the strength of the singularity, but not its existence. 


\section{COMMUTATIVITY IN FULL S $\chi$ PT}

The lack of commutativity of limits appears in the computed one-loop S $\chi \mathrm{PT}$ quantities 7, 8, 11] only in the quenched or partially quenched cases, which are enhanced in the IR relative to the full case and therefore especially sensitive to the chiral limit. This is due to the double pole structure that shows up in flavor-neutral meson propagators. In the full QCD case, there are only single poles, and I therefore do not expect noncommutativity to be generic in this case. For example, if we first go to full QCD $\left(m_{V}=m_{S} \equiv m ; \chi_{V V}=\chi_{S S} \equiv \chi\right)$ in Eqs. (5) and (6), the NLO corrections then always vanish in the subsequent $m \rightarrow 0, a \rightarrow 0$ limits, independent of their order.

The absence of the commutativity problem in most full QCD quantities seems to be a general feature of $\mathrm{S} \chi \mathrm{PT}$. It is equivalent to the statement that the limit $m \rightarrow 0$ at fixed $a$ is smooth in full $\mathrm{S} \chi \mathrm{PT}$, i.e., that there are no IR divergences on shell in this limit. I believe this to be the case because, taking the additional limit $a \rightarrow 0$, one recovers the massless continuum $\chi \mathrm{PT}$. Ordinary chiral power counting in this limit shows that IR divergences are absent: The derivative couplings suppress IR contributions and make loop effects finite even in the presence of massless particles. ${ }^{2}$ If we now turn on the lattice-dependent $\left(\mathcal{O}\left(a^{2}\right)\right.$ and higher) vertices of $\mathrm{S} \chi \mathrm{PT}$, these act as effective mass terms and should not induce IR divergences that were previously absent. Power counting applied to S $\chi \mathrm{PT}$ then implies that any logarithms of $a^{2}$ that appear in the $m \rightarrow 0$ limit are "protected" by powers of $a^{2}$, so they cause no problem in the subsequent $a \rightarrow 0$ limit.

Full $\mathrm{S} \chi \mathrm{PT}$ in the chiral limit is in fact closely analogous to full continuum $\chi \mathrm{PT}$ with massless up and down quarks but a nonvanishing strange quark mass. In both cases there are terms giving mass to some, but not all, of the pseudoscalar mesons: The pions remain massless in the continuum example; while the taste- $\xi_{5}$ (Goldstone) meson remains massless in $\mathrm{S} \chi \mathrm{PT}$. And in both cases the mass or mass-like terms give rise to interactions lacking enough derivatives to suppress all IR divergences automatically. Yet, one expects no IR divergences in the continuum case as the strange quark mass turns on, and therefore I expect $\mathrm{S} \chi \mathrm{PT}$ to be likewise well-behaved.

However, a detailed proof that the chiral limit of full S $\chi \mathrm{PT}$ is "safe" may be rather

\footnotetext{
${ }^{2}$ Note that one needs to go on shell so that derivatives acting on external lines also suppress the diagrams in the massless limit.
} 
delicate. Although the lattice-dependent vertices give no nonderivative interactions purely among the taste- $\xi_{5}$ mesons [6, 7, 10] (the analogous statement is also true in the continuum example), potential problems may arise when external non-Goldstone mesons (taste different from $\left.\xi_{5}\right)$ scatter to give massless $\xi_{5}$ mesons in internal loops.

As a simple example, consider massless S $\chi$ PT theory with two flavors, and take only the coefficient $C_{4}$ in the taste-violating, $\mathcal{O}\left(a^{2}\right)$, potential [6, 7] to be nonzero. This is sufficient to give mass to all the non-Goldstone mesons. Now consider the contribution shown in Fig. 11 to the scattering of two taste-singlet, flavor charged, mesons through the exchange of taste- $\xi_{5}$ mesons. If the two vertices come from the $C_{4}$ term, then there are no derivatives on the internal lines to suppress the logarithmic IR divergence. On shell, the momentum transfer through the loop will, in the generic case, provide a cutoff. However, with $C_{4}$ vertices, there is an IR divergence in this diagram in the forward direction, $p_{1}=p_{3}, p_{2}=p_{4}$. The divergence is canceled by the diagrams with one or both of the vertices replaced by kinetic energy vertices. The terms in the kinetic energy vertices where the derivatives act on the external lines provide the cancellation, possible because $p_{1}=p_{3}$ implies $p_{1} \cdot p_{3}=-a^{2} \Delta_{I}=-64 a^{2} C_{4} / f^{2}$. Terms where the derivatives act on the internal lines are clearly not IR divergent; neither is the corresponding "s-channel" diagram (on shell). Though the result of this example is positive (no IR divergence on shell), it also shows some of the issues that would need to be addressed in a complete proof that full $\mathrm{S} \chi \mathrm{PT}$ is safe.

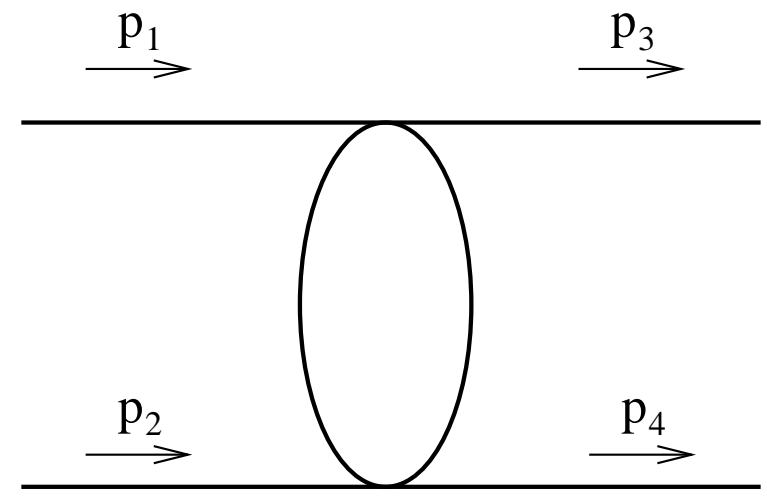

FIG. 1: A "t-channel" scattering diagram for the scattering of two taste-singlet mesons, with internal taste- $\xi_{5}$ mesons: $\pi_{I}^{+}\left(p_{1}\right)+\pi_{I}^{-}\left(p_{2}\right) \rightarrow \pi_{I}^{+}\left(p_{3}\right)+\pi_{I}^{-}\left(p_{4}\right)$.

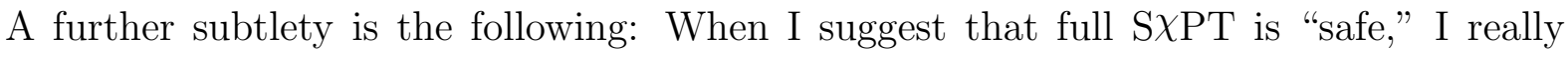
only mean that certain standard quantities are safe, i.e., they have commuting chiral and 
continuum limits. A sufficient number of derivatives (with respect to quark mass) of any safe quantity will certainly produce IR divergences and hence lack of commutativity of limits. Which quantities are likely to be safe? The above arguments suggest that quantities that are free of IR divergences in the chiral limit of full continuum $\chi \mathrm{PT}$ will probably be safe in $\mathrm{S} \chi \mathrm{PT}$. This means, for example, that pseudoscalar masses and decay constants as computed in 2] will be safe. On the other hand, a quantity like the pion charge radius, ${ }^{3}$ which is defined in terms of an external current with nonvanishing momentum transfer, is IR divergent in the continuum chiral limit [14]. The charge radius is therefore almost certain to have the wrong chiral limit at fixed $a$ in $\mathrm{S} \chi \mathrm{PT}$; it should indeed behave much the same way as the quenched $M_{\pi}^{2} / 2 \mu m_{V}$, Eqs. (11) and (2).

Furthermore, since in the continuum one can differentiate the squared meson mass once with respect to the quark mass and still have a finite quantity in the chiral limit, I expect that the corresponding quantities in $\mathrm{S} \chi \mathrm{PT}$ are safe. For the squared Goldstone (taste $\xi_{5}$ ) mass this is fairly obvious from the exact lattice $U_{A}(1)$; one can also divide by (instead of differentiating with respect to) the quark mass, as in the full $\mathrm{S} \chi \mathrm{PT}$ version of Eq. (6). It is less obvious that one can differentiate the squared masses of non-Goldstone (taste other than $\left.\xi_{5}\right)$ mesons and still have a safe quantity. For example, how do we know that a term like $a^{2} \chi \ln \chi$ cannot appear in the squared non-Goldstone masses? Such terms are not divergent as $m \rightarrow 0$, but their derivatives are divergent, which would lead to noncommutativity. A one-loop calculation, similar to the scattering calculation described above, shows that such terms do in fact cancel for the taste-singlet mass. Whether this really persists beyond one loop remains to be seen.

Note that dividing a squared non-Goldstone mass by quark mass, rather than differentiating, is certainly unsafe, as is already clear at tree-level, Eq. (31). This shows why it is difficult to write down a simple rule that distinguishes safe from unsafe quantities in all cases. On the other hand, if we limit ourselves only to Goldstone mesons on the external

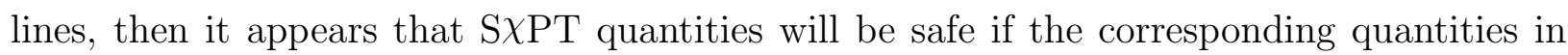
full continuum $\chi \mathrm{PT}$ are finite in the chiral limit.

\footnotetext{
${ }^{3}$ I thank S. Sharpe for this example
} 


\section{ERRORS OF EXISTING NUMERICAL COMPUTATIONS}

Dürr and Hoelbling [1] worry that the possible noncommutativity of limits with staggered fermions would induce new, uncontrolled, errors in the previously reported results with MILC staggered configurations [2, 3, 4, 5]. To the extent that S $\chi \mathrm{PT}$ describes the MILC simulations, we can argue that such errors are not present - even in the absence of a detailed proof that full S $\chi \mathrm{PT}$ is safe to all orders. That is because the extraction of physical results requires extrapolation only to the physical light quark masses, not to the chiral limit. The factor $\ln \left(\chi / \Lambda^{2}\right)$ is of order 3 or 4 at the physical point $(\chi \approx 140 \mathrm{MeV})$. Even should the logarithm not be "protected" by powers of $\chi$ in some higher order of S $\chi \mathrm{PT}$ and represent a divergence in the chiral limit at fixed $a$, it would still be suppressed, numerically, by the additional powers of $a^{2}$ at that order. By looking at the numerics of the calculations in Refs. [2, 3, 4, 5], it is not hard to convince oneself that any new errors would most likely be significantly smaller than the already-quoted systematic errors.

As an example, consider the case of $f_{\pi}$. Imagine that there is in fact an "unsafe" contribution at NNLO (next-to-next-to-leading order), i.e., of order $a^{4} \ln \left(\chi / \Lambda^{2}\right)$. To estimate its size, define the dimensionless taste-violating chiral expansion parameter $x_{a^{2}}$ by [2]

$$
x_{a^{2}} \equiv \frac{a^{2} \bar{\Delta}}{8 \pi^{2} f_{\pi}^{2}},
$$

where $a^{2} \bar{\Delta}$ is a "typical" taste-violating term at $\mathcal{O}\left(a^{2}\right)$. Taking for $a^{2} \bar{\Delta}$ the average pion splitting, gives $x_{a^{2}} \approx 0.09$ on the MILC coarse lattices and $x_{a^{2}} \approx 0.03$ on the fine lattices.

Then I would expect the putative NNLO unsafe contribution to be generated by a term of size

$$
\left(x_{a^{2}}\right)^{2} \frac{1}{16} \sum_{B} \ln \chi_{B} / \Lambda^{2},
$$

where the sum is over all 16 pion tastes, labeled by $B$. The unsafe contribution comes from the Goldstone pion in the sum. Note that I assume that the Goldstone pion appears only through such an average over tastes. That seems very likely, since the diagrams that distinguish separate tastes are diagrams with disconnected internal pion propagators, which at NLO occur just for the singlet, axial, and vector tastes. At NNLO there could be disconnected internal Goldstone lines, but it is known [10] that such contributions come with an extra factor of $p^{2}$, making them safe. Putting in $\ln \chi / \Lambda^{2} \approx 4$ and the values of $x_{a^{2}}$ gives an unsafe contribution of $\approx 0.2 \%$ on the coarse lattices and $\approx 0.02 \%$ on the fine lattices. These 
are clearly much smaller than other systematic errors (which are $\approx 3 \%$ for $f_{\pi}$ and $\approx 1 \%$ for $\left.f_{K} / f_{\pi}\right)$, even without taking into account the fact that some of the error would be removed by the continuum extrapolation (linear in $\alpha_{S}^{2} a^{2}$ in Ref. [2]).

Of course, there would also be systematic errors coming from contributions of the other taste pions in Eq. (12). However, these contributions are safe, with commuting chiral and continuum limits. The associated errors are therefore standard higher order S $\chi \mathrm{PT}$ errors, and as such are already included in the error estimates of Ref. [2].

Note that my error estimate assumes that the "worst case" would be the existence of a singularity in full $\mathrm{S} \chi \mathrm{PT}$ that would interfere with the extrapolation to the physical light quark masses. This is reasonable despite the fact that intermediate stages in the analysis in Refs. 22, 3, 4, 5] often involve fits to partially quenched lattice data, where we know that noncommutativity of limits does occur. The point is that the relevant NLO formulas such as Eq. (6) are known. We fit lattice data to them in a range of valence and sea masses that are far from the singularities. Since the light quark masses in the data are always more than a factor of three larger than the physical masses, the logarithms and the nonlinearities associated with them are always significantly smaller than at the physical point. It is therefore not surprising that (as checked directly in the calculations) the NLO corrections in the fits are under control and of the expected magnitude. If unexpected new singularities were to occur in higher order, the resulting errors in the partially quenched fits would be smaller than those induced in the subsequent full S $\chi$ PT extrapolation, simply because the logarithms are smaller.

Of course, if the staggered simulations had some additional noncommutativity of limits not captured by $\mathrm{S} \chi \mathrm{PT}$ - having to do, say, with the fourth-root procedure - then an uncontrolled systematic error might in fact be present in the MILC results. However, recent advances in understanding the rooting procedure [15, 16, 17], coupled with the good fit of MILC data [2] to S $\chi \mathrm{PT}$ predictions [7, 8], make such a possibility seem increasingly unlikely.

\section{ADDITIONAL REMARKS}

The lack of commutativity of the chiral and continuum limits is not exclusively a property of staggered fermions. For Wilson fermions, it appears at one loop in a power counting for which $\mathcal{O}(m)$ and $\mathcal{O}\left(a^{2}\right)$ are treated as comparable [18]. At this order, a term of the form 
$a^{2} \chi \ln \chi$ is present in the squared meson mass in the full QCD case. ${ }^{4}$ (The corresponding partially quenched case has not to my knowledge been studied.) After differentiating with respect to (or dividing by) the quark mass, such a term leads to an IR divergence as $m \rightarrow 0$ for fixed $a$; whereas it clearly vanishes if the $a \rightarrow 0$ limit is taken first. The source of problem is the absence of an exact chiral symmetry in the massless limit. This means that massless Wilson pions can interact without derivative couplings [19], leading to IR singularities and failure of commutativity.

Note that the Wilson case is significantly worse than the staggered case: In the latter, self-interactions of the massless Goldstone (taste $\xi_{5}$ ) pion are always proportional to at least two powers of momentum. At finite $a$, the non-Goldstone staggered pions are not required to have derivative interactions, but neither are they massless.

My S $\chi \mathrm{PT}$ examples and certainly the Wilson case suggest that lack of commutativity has little or nothing to do with the rooting procedure. Is this also true of the 2D Schwinger models studied in Ref. [1] ? The physics of the one-flavor 2D Schwinger model $\langle\bar{\psi} \psi\rangle$, where the noncommutativity is found, is quite distinct from the multiflavor $4 \mathrm{D}$ chiral theories discussed above. With one flavor, the "condensate" comes about because of the symmetry is violated by the anomaly, not because of spontaneous symmetry breaking. In the continuum, the anomaly leads to exact zero modes of the Dirac operator, which in turn saturate $\langle\bar{\psi} \psi\rangle$. Since staggered fermions have only near zero modes in complex pairs, their effect cancels in the $m \rightarrow 0$ limit, and $\langle\bar{\psi} \psi\rangle$ vanishes for fixed $a$. If the continuum limit is taken first, however, the complex pair of near zero modes becomes a pair of degenerate exact zero modes, and the rooting prescription works as desired, producing a single exact mode. (See Refs. [1, 20] for details.) Since the physics here is inextricably tied to having only one flavor, however, it is not possible to separate cleanly the issues of the lack of commutativity and the rooting procedure within this model.

In the two-flavor 2D Schwinger model, on the other hand, the rooting procedure is not required, yet I suspect that it would be possible to find noncommutativity in the masses of the "quasi-Goldstone bosons," of a similar nature to that seen in quenched or partially quenched 4D S $\chi \mathrm{PT}$ (Sec. 【II). That is because integration over a single (boson) pole in 2D has the same IR behavior as integration over a double pole in $4 \mathrm{D}$. The problem here is

\footnotetext{
${ }^{4}$ I am grateful to O. Bär for pointing out to me this result in Ref. [18].
} 
that there is no true condensate in $2 \mathrm{D}$, so there is no simple chiral theory: $\langle\bar{\psi} \psi\rangle$ and the quasi-Goldstone boson squared masses vanish as fractional powers of $m$ as $m \rightarrow 0$ [21]. It is therefore a nontrivial problem to discover an analogue of $\mathrm{S} \chi \mathrm{PT}$ that would allow one to calculate the discretization effects caused by staggered fermions. Thus I am not able at this point to make a specific proposal for where to look for noncommutativity in this model.

A simple alternative to the two-flavor theory in this context can be found in the quenched 2D Schwinger model, which does not require the rooting procedure either (trivially). Reference [20] studies the quenched theory with staggered fermions, but since $\langle\bar{\psi} \psi\rangle$ blows up as $1 / m$ in the continuum, it is hard to see any noncommutativity cleanly. Recently, Dürr and Hoelbling have looked instead at $m\langle\bar{\psi} \psi\rangle / g^{2}$ ( $g$ is the coupling) for staggered fermions 22]. They find clear evidence that the chiral and continuum limits do not commute for this quantity. In addition, the behavior is is very similar, qualitatively, to that observed for $\langle\bar{\psi} \psi\rangle$ in the one-flavor Schwinger model. At the least, this shows that noncommutativity of limits is not inextricably tied to the rooting procedure in these $2 \mathrm{D}$ models.

Finally, one may wonder whether there are order-of-limits problems in 4D that are directly analogous to those in the one-flavor 2D Schwinger model, and not of the kind treated above in $\mathrm{S} \chi \mathrm{PT}$. For example, in QCD with a single flavor, the value of $\langle\bar{\psi} \psi\rangle$ in the chiral limit is probably crucially dependent on having exact zero modes [20], just as it is in 2D, leading to noncommuting chiral and continuum limits. In normal, multiflavor QCD, however, I know of no standard physical quantities that have this kind of sensitivity to the existence of exact zero modes. For example, the topological susceptibility seems to be well-behaved, both in $\mathrm{S} \chi \mathrm{PT}[9]$ and in simulations [23]. This is not to say that the staggered discretization errors are negligible — indeed they are large. But there is no commutativity problem; the continuum suppression of the susceptibility seems to be present whether one takes the chiral or continuum limit first. Similarly, I do not expect any problem in the QCD $\eta^{\prime}$ mass. Because of the anomaly, the $\eta^{\prime}$ mass is generated through disconnected meson diagrams [24]. In the continuum limit, staggered fermions have the correct anomaly (for infinitesimal chiral rotations) [25], and this should be adjusted correctly by the fourth-root procedure, since the anomaly is essentially perturbative. At finite lattice spacing, I expect that S $\chi \mathrm{PT}$ will correctly capture the modifications in the $\eta^{\prime}$ physics due to staggered fermions, at least at 
vacuum angle $\theta=0,{ }^{5}$ just as ordinary $\chi \mathrm{PT}$ correctly captures $\eta^{\prime}$ physics in the continuum [28].

\section{ACKNOWLEDGMENTS}

I am very grateful to Stephan Dürr and Christian Hoelbling for extensive discussions and for communicating their results on the quenched Schwinger model before publication. I also thank Oliver Bär, Maarten Golterman, Jim Hetrick, Gautam Rupak, and Steve Sharpe for many helpful comments and suggestions. Finally, I am grateful to Peter Weisz for pointing out the misleading description of "standard quantities" in version 2 of this paper. This work is partially supported by the US Department of Energy under grant DE-FG02-91ER40628.

[1] S. Dürr and C. Hoelbling, Phys. Rev. D 71, 054501 (2005) arXiv:hep-lat/0411022.

[2] C. Aubin et al. (MILC), Phys. Rev. D 70, 114501 (2004) arXiv:hep-lat/0407028.

[3] C. Aubin et al. (HPQCD, MILC, UKQCD), Phys. Rev. D 70, 031504(R) arXiv:hep-lat/0405022.

[4] C. Aubin et al. (MILC), Phys. Rev. D 70, 094505 (2004) arXiv:hep-lat/0402030.

[5] C. T. H. Davies et al. (Fermilab, HPQCD, MILC, UKQCD), Phys. Rev. Lett. 92, 022001 (2004) arXiv:hep-lat/0304004.

[6] W. J. Lee and S. R. Sharpe, Phys. Rev. D 60, 114503 (1999) arXiv:hep-lat/9905023; C. Bernard, Phys. Rev. D 65, 054031 (2002) arXiv:hep-lat/0111051.

[7] C. Aubin and C. Bernard, Phys. Rev. D 68, 034014 (2003) arXiv:hep-lat/0304014.

[8] C. Aubin and C. Bernard, Phys. Rev. D 68, 074011 (2003) arXiv:hep-lat/0306026.

[9] B. Billeter, C. DeTar and J. Osborn, Phys. Rev. D 70, 077502 (2004) arXiv:hep-lat/0406032.

[10] S. R. Sharpe and R. S. Van de Water, arXiv:hep-lat/0409018.

\footnotetext{
${ }^{5}$ Away from $\theta=0$, the issue is not simply one of commutativity of limits, but whether staggered fermions can get the correct physics at all. This is related to the fact that the staggered anomalous symmetry involves a lattice translation and cannot be exponentiated to trade a phase in the quark mass matrix for $\theta$. For example, the known CP-violating phase [26] cannot be reproduced by a simulation with an odd number of negative mass staggered quarks 27$]$.
} 
[11] C. Aubin and C. Bernard, Nucl. Phys. B (Proc. Suppl.) 140, 491 (2005) arXiv:hep-lat/0409027, and in preparation.

[12] C. Bernard and M. Golterman, Phys. Rev. D 46, 853 (1992); S.R. Sharpe, Phys. Rev. D 46, 3146 (1992).

[13] S. R. Sharpe, Phys. Rev. D 56, 7052 (1997) [Erratum-ibid. D 62, 099901 (2000)] arXiv:hep-lat/9707018.

[14] M. A. B. Beg and A. Zepeda, Phys. Rev. D 6, 2912 (1972); M. K. Volkov and V. N. Pervushin, Yad. Fiz. 19, 652 (1974); J. Gasser and H. Leutwyler, Annals Phys. 158, 142 (1984).

[15] F. Maresca and M. Peardon, arXiv:hep-lat/0411029.

[16] D. H. Adams, arXiv:hep-lat/0411030.

[17] Y. Shamir, arXiv:hep-lat/0412014.

[18] S. Aoki, Phys. Rev. D 68, 054508 (2003) arXiv:hep-lat/0306027.

[19] N. Kawamoto and J. Smit, Nucl. Phys. B 192, 100 (1981).

[20] S. Dürr and C. Hoelbling, Phys. Rev. D 69, 034503 (2004) arXiv:hep-lat/0311002.

[21] S. Coleman, Annals Phys. 101, 239 (1976); A. V. Smilga, Phys. Lett. B 278, 371 (1992);

J. E. Hetrick, Y. Hosotani and S. Iso, Phys. Lett. B 350, 92 (1995) arXiv:hep-th/9502113.

[22] S. Dürr and C. Hoelbling, private communication.

[23] C. Aubin et al. (MILC), Nucl. Phys. B (Proc. Suppl.) 140, 600 (2005) arXiv:hep-lat/0409051;

C. Bernard et al. (MILC), Phys. Rev. D 68, 114501 (2003) arXiv:hep-lat/0308019.

[24] E. Witten, Nucl. Phys. B 156, 269 (1979).

[25] H. S. Sharatchandra, H. J. Thun and P. Weisz, Nucl. Phys. B 192, 205 (1981).

[26] R. Dashen, Phys. Rev. D 3, 1879, (1971); E. Witten, Annals. of Phys. 128, 363 (1980).

[27] M. Creutz, Phys. Rev. Lett. 92, 201601 (2004) arXiv:hep-lat/0312018; I. Montvay, private communication.

[28] J. Gasser and H. Leutwyler, Nucl. Phys. B 250, 465 (1985). 